\title{
ON THE GORENSTEIN PROPERTY OF REES AND FORM RINGS OF POWER OF IDEALS
}

\author{
M. HERRMANN, J. RIBBE, AND S. ZARZUELA \\ Dedicated to Professor H. Hironaka on occasion of his 60 th birthday
}

\begin{abstract}
In this paper we determine the exponents $n$ for which the Rees ring $R\left(I^{n}\right)$ and the form ring $\operatorname{gr}_{A}\left(I^{n}\right)$ are Gorenstein rings, where $I$ is a strongly Cohen-Macaulay ideal of linear type (including complete and almost complete intersections) or an $\mathfrak{m}$-primary ideal in a local ring $A$ with maximal ideal $\mathfrak{m}$.
\end{abstract}

Given an ideal $I$ in a local ring $(A, \mathfrak{m})$ it is well known that the CohenMacaulayness of the Rees algebra $R(I)$ implies the Cohen-Macaulayness of all Rees algebras $R\left(I^{n}\right)$. The same is true for the form rings $\operatorname{gr}_{A}(I)$ and $\operatorname{gr}_{A}\left(I^{n}\right)$; see $[3,(2.7 .8)$ and $(8.8 .5)]$. In this paper we show that, in contrast to the CohenMacaulay property, the Gorenstein property of $R\left(I^{n}\right)$ and $\operatorname{gr}_{A}\left(I^{n}\right)$ only holds for special exponents $n$. If in particular $\operatorname{gr}_{A}(I)$ is Gorenstein and $R(I)$ is Cohen-Macaulay it turns out that these special exponents are closely related to the $a$-invariant of the form ring $\operatorname{gr}_{A}(I)$. Mainly under this aspect we prove some results concerning the Gorenstein property of Rees and form rings of powers of

(i) strongly Cohen-Macaulay ideals of linear type (including almost complete intersections) in Gorenstein rings,

(ii) m-primary ideals in Cohen-Macaulay rings, and

(iii) equimultiple prime ideals $\mathfrak{p} \neq \mathfrak{m}$ in a generalized Cohen-Macaulay ring.

Our investigations are essentially based on the explicit computation of the $a$-invariants of form (and Rees) rings (see $\S 2$ ). For the above classes of idealsusing a structure theorem for the canonical module of $R(I)$ in [7]-we can determine in $\S 3$ the exponents $n \geq 1$ for which $R\left(I^{n}\right)$ and $\operatorname{gr}_{A}\left(I^{n}\right)$ are Gorenstein rings (see in particular Theorem (3.5)). A more geometrical interpretation of the results in $\S 3$ is the observation that in these situations the Gorenstein property of $\operatorname{Proj}(R(I))$ can be deduced from the Gorenstein property of a certain Veronesean subring $R\left(I^{n}\right)$ of $R(I)$. It might be an interesting question when the Gorenstein property of a blow up $\operatorname{Proj}(R(I))$ is inherited to an appropriate Rees ring $R(J)$ with $\operatorname{Proj}(R(J))=\operatorname{Proj}(R(I))$ over $\operatorname{Spec} A$.

In $\S 4$, Part I we characterize-for Cohen-Macaulay (or Gorenstein) rings $A$-the Gorenstein property of $R\left(\mathrm{~m}^{d-i}\right), i=1,2,3$, by conditions on the

Received by the editors June 30, 1991 and, in revised form, April 10, 1992.

1991 Mathematics Subject Classification. Primary 13D03, 13H10, 13H15; Secondary 14B05, $14 \mathrm{~B} 15$. 
reduction exponent of $\mathfrak{m}$ (see Theorem (4.4)). In Part II we describe sufficient conditions on an equimultiple prime ideal $\mathfrak{p}$ in $A$ for being a complete intersection in terms of blow up properties. In case that $\operatorname{dim}(A) \geq 2 \mathrm{ht}(\mathfrak{p})$ and $A$ is a generalized Cohen-Macaulay ring, the Gorenstein property of $R\left(\mathfrak{p}^{\mathrm{ht}(\mathfrak{p})-1}\right)$ implies that $\mathfrak{p}$ is a complete intersection $(\mathrm{ht}(a)$ denotes the height of an ideal $a$ ). For a somewhat similar question for projective schemes we refer to the forthcoming paper [16].

Some of the results in $\S 4$ were also proved independently by A. Ooishi $[10,11]$ by different methods. We would like to thank A. Ooishi for many stimulating discussions during the preparation of this work.

\section{Preliminaries}

To study the relationship between the various graded rings associated to an ideal $I$ in a (commutative) Noetherian ring $A$, we will also use the so-called approximation complexes of $I$ (see $[4,5$, and 6]). The situation is particularly good when these complexes are exact, a condition that is fulfilled for some important families of ideals. In these cases it is often possible to prove that those graded rings are Cohen-Macaulay or Gorenstein. One important point in this theory is that under the "good" situation the ideal $I$ is of linear type, namely $\operatorname{Sym}_{A}(I) \simeq R(I)$.

Assume for simplicity that $A$ is a local ring with maximal ideal $\mathfrak{m}$ and $\operatorname{dim}(A)=d$. Let $I$ be an ideal of $A$ and $\underline{a}:=\left\{a_{1}, \ldots, a_{n}\right\}$ a system of generators of $I$, and consider the Koszul complex $K(\underline{a})$ of $A$ w.r.t. $\underline{a}$. If we denote by $S$ the polynomial ring $A\left[X_{1}, \ldots, X_{n}\right]$ it is possible to get two complexes of $S$-modules:

$$
\mathscr{Z}(\underline{a}): 0 \rightarrow \mathscr{Z}_{n} \rightarrow \cdots \rightarrow \mathscr{Z}_{1} \rightarrow \mathscr{Z}_{0} \rightarrow 0
$$

and

$$
\mathscr{M}(\underline{a}): 0 \rightarrow \mathscr{M}_{n} \rightarrow \cdots \rightarrow \mathscr{M}_{1} \rightarrow \mathscr{M}_{0} \rightarrow 0,
$$

where $\mathscr{Z}_{i}=Z_{i}(K(\underline{a})) \otimes_{A} S$ and $\mathscr{M}_{i}=H_{i}(K(\underline{a})) \otimes_{A} S$.

The $Z_{i}(K(\underline{a}))$ denote the cycles of the Koszul complex $K(\underline{a})$, and the $H_{i}(K(\underline{a}))$ denote the Koszul homology. Both complexes can be taken as complexes of graded modules over $S$ with mappings of degree -1 .

We list the main properties of these complexes:

(1) The homology of $\mathscr{Z}(\underline{a})$ and $\mathscr{M}(\underline{a})$ is independent of the system of generators $\underline{a}$.

(2) $H_{0}(\mathscr{Z}(\underline{a}))=\operatorname{Sym}_{A}(I)$.

(3) $H_{0}(\mathscr{M}(\underline{a}))=\operatorname{Sym}_{A}\left(I / I^{2}\right)$.

(4) The following are equivalent:

(i) $\mathscr{M}(\underline{a})$ is acyclic,

(ii) $\mathscr{Z}(\underline{a})$ is acyclic and $I$ is of linear type.

Now assume that $A$ is Cohen-Macaulay. Then the following properties (5) to (9) hold:

(5) Suppose:

(i) For any prime ideal $\mathfrak{p} \supseteq I, \mu\left(I_{\mathfrak{p}}\right) \leq \mathrm{ht}(\mathfrak{p})$ (where $\mu(-)$ denotes the minimal number of generators).

(ii) For any $r \geq 0$ and for any prime ideal $\mathfrak{p} \supseteq I$,

$$
\operatorname{depth}_{A \mathfrak{p}}\left(H_{r}(K(\underline{a}))_{\mathfrak{p}}\right) \geq \inf (r, \operatorname{ht}(\mathfrak{p} / I)) .
$$

Then $\mathscr{M}(\underline{a})$ is acyclic. 
It turns out that the condition (ii) above is independent of the system of generators of $I$, and that it is fulfilled if $I$ is strongly Cohen-Macaulay (sCM for short), that is, if for any $r \geq 0$ the Koszul homology $H_{r}(K(\underline{a}))$ is zero or a maximal Cohen-Macaulay $A / I$-module.

(6) Suppose:

(i) For any prime ideal $\mathfrak{p} \supseteq I, \mu\left(I_{\mathfrak{p}}\right) \leq \mathrm{ht}(\mathfrak{p})+1$.

(ii) For any $r \geq 0$ and for any prime ideal $\mathfrak{p} \supseteq I$,

$$
\operatorname{depth}_{A \mathfrak{p}}\left(H_{r}(K(\underline{a}))_{\mathfrak{p}}\right) \geq \inf (r, \operatorname{ht}(\mathfrak{p} / I))-1 .
$$

Then $\mathscr{Z}(\underline{a})$ is acyclic.

(7) Assume that $\mathrm{ht}(I) \geq 1$ and

(i) For any prime ideal $\mathfrak{p} \supseteq I, \mu\left(I_{\mathfrak{p}}\right) \leq \mathrm{ht}(\mathfrak{p})$,

(ii) $I$ is strongly Cohen-Macaulay.

Then $\operatorname{gr}_{A}(I)$ and $R(I)$ are Cohen-Macaulay. Moreover, if $A$ is Gorenstein then $\operatorname{gr}_{A}(I)$ is Gorenstein.

There is an important connection between the theory of approximation complexes and the theory of $d$-sequences:

(8) If $I$ is generated by a $d$-sequence, then $\mathscr{M}(\underline{a})$ is acyclic.

(9) If $|A / \mathfrak{m}|=\infty$ and $\mathscr{M}(\underline{a})$ is acyclic, then $I$ can be generated by a $d$-sequence (but the given $\underline{a}$ is not necessarily a $d$-sequence!).

Finally we mention some relevant families of strongly Cohen-Macaulay ideals:

(a) Complete intersection ideals.

(b) Ideals $I \subseteq A$ such that $\mu(I)=\mathrm{ht}(I)+1$ and $A / I$ is Cohen-Macaulay. In particular, almost complete intersection (a.c.i) ideals $I$ such that $A / I$ is CohenMacaulay are sCM. We can say that an ideal $I$ is a.c.i. if $\mu(I)=\mathrm{ht}(I)+1$ and $I A_{\mathfrak{p}}$ is complete intersection for any prime ideal $\mathfrak{p} \in \operatorname{Min}(A / I)$.

(c) Suppose $A$ is Gorenstein and $I \subset A$ is an ideal such that $\mu(I)=\mathrm{ht}(I)+2$. If $A / I$ is Cohen-Macaulay then $I$ is $\mathrm{sCM}$.

\section{THE $a$-INVARIANT OF GRADED MODULES}

In this section we collect some important properties of the $a$-invariants of Rees and form rings.

Recall that for a positively graded Noetherian ring $R=\bigoplus_{i \geq 0} R_{i}$ defined over a local ring $R_{0}$ and a Noetherian graded $R$-module $G$ the $a$-invariant of $G$ is defined as

$$
a(G)=\max \left\{j \in Z: H_{M}^{r}(G)_{j} \neq 0\right\},
$$

where $r=\operatorname{dim}(G)$ and $H_{M}^{r}(G)$ is the $r$ th local cohomology w.r.t. the maximal homogeneous ideal $M$ of $R$. Note that $R$ is Gorenstein if and only if $R$ is Cohen-Macaulay and the canonical module of $R$ is $K_{R} \simeq R(a(R))$, see [3, Chapter VII].

We start with an easy but useful lemma. For completeness we sketch a proof.

Lemma (2.1). Let $R=\bigoplus_{n \geq 0} R_{n}$ be a Noetherian graded algebra defined over a local ring $R_{0}$ and $G=\bigoplus_{n \geq 0} G_{n}$ a Noetherian graded $R$-module. Let $x \in R_{1}$ be a regular element on $G$. Then we get for the a-invariants of $G$ and $G / x G$

$$
a(G) \leq a(G / x G)-1 .
$$

Moreover, if $G$ is Cohen-Macaulay the equality holds. 
Proof. Consider the exact sequence of graded modules

$$
0 \rightarrow G(-1) \stackrel{\cdot x}{\rightarrow} G \rightarrow G / x G \rightarrow 0 .
$$

Putting $r=\operatorname{dim}_{R}(G)$ and $M=$ maximal homogeneous ideal of $R$, we get from (1) the long exact cohomology sequence

$$
\cdots \rightarrow H_{M}^{r-1}(G)_{i} \rightarrow H_{M}^{r-1}(G / x G)_{i} \rightarrow H_{M}^{r}(G)_{i-1} \rightarrow H_{M}^{r}(G)_{i} \rightarrow 0 .
$$

For $i=a(G)+1$ we have

$$
H_{M}^{r}(G)_{i-1} \neq 0 \text { and } H_{M}^{r}(G)_{i}=0 .
$$

Then (2) implies $H_{M}^{r-1}(G / x G)_{i} \neq 0$, i.e. $a(G / x G) \geq i=a(G)+1$. This proves the first part of the claim. The second part follows from (2) since now $H_{M}^{r-1}(G)=0$.

In our context the importance of $a$-invariants is a consequence of the following characterizations of the Cohen-Macaulay and Gorenstein property of Rees algebras.

Proposition (2.2). Let I be an ideal of positive height in a d-dimensional local ring $(A, \mathfrak{m})$.

(a) [15, Theorem 1.1] The Rees algebra $R(I)$ is a Cohen-Macaulay ring if and only if

$$
H_{M}^{i}\left(\operatorname{gr}_{A}(I)\right)_{n}=0 \text { for } n \neq-1, i=0, \ldots, d-1,
$$

and

$$
\left.H_{M}^{d}\left(\operatorname{gr}_{A}(I)\right)_{n}=0 \text { for } n \geq 0 \text { (i.e. } a\left(\operatorname{gr}_{A}(I)\right)<0\right),
$$

where $M$ denotes the maximal homogeneous ideal of $R(I)$. In this case, $H_{M}^{i}\left(\operatorname{gr}_{A}(I)\right)_{-1} \simeq H_{\mathfrak{m}}^{i}(A)$ for $i=0, \ldots, d-1$.

(b) [8, Theorem (3.1)] If $R(I)$ is Cohen-Macaulay and grade $(I) \geq 2$, then $R(I)$ is Gorenstein if and only if $a\left(\mathrm{gr}_{A}(I)\right)=-2$ and the rings $A$ and $\mathrm{gr}_{A}(I)$ are quasi-Gorenstein (i.e. the canonical modules of the specific rings are isomorphic to the suitably shifted rings).

Remark (2.3). While for any nonpositive integer $a$ there is a local ring $A$ and an ideal $I \subseteq A$ with $a=a\left(\mathrm{gr}_{A}(I)\right)$, the $a$-invariant of the Rees algebra $R(I)$ is in many cases strictly determined. In particular if $R(I)$ is Cohen-Macaulay and $\operatorname{ht}(I)>0$, then $a(R(I))=-1$. In this case $a\left(\operatorname{gr}_{A}(I)\right) \leq-1$. This can be easily deduced from the proof of Proposition (2.1) in [8].

For several classes of ideals $I$ the $a$-invariant of the form ring $\mathrm{gr}_{A}(I)$ can be explicitly computed. In the following Lemma (2.4) we recall some well-known facts concerning m-primary ideals, and in Proposition (2.5) we determine the $a$-invariant $a\left(\operatorname{gr}_{A}(I)\right)$ for a strongly Cohen-Macaulay ideal which is of linear type. First we define the reduction exponent $\delta(I)$ of an ideal $I$ in a local ring $A$ with infinite residue field as

$$
\delta(I):=\min \left\{n \in N \mid \text { there exists a minimal reduction } q \text { of } I \text { s.t. } I^{n+1}=q I^{n}\right\} .
$$

Lemma (2.4). Let $I$ be an m-primary ideal in a local ring $(A, \mathfrak{m})$. Then the following hold: 
(a) [13, Theorem and Theorem 2.1] If the reduction exponent of the maximal ideal $\mathfrak{m}$ of $A$ is 0,1 or 2 , then $\mathrm{gr}_{A}(\mathfrak{m})$ is Cohen-Macaulay (resp. Gorenstein) iff $A$ is so.

(b) If $\operatorname{gr}_{A}(I)$ is Cohen-Macaulay, then

$$
a\left(\operatorname{gr}_{A}(I)\right)=\delta(I)-\operatorname{dim}(A) .
$$

Statement (b) follows from (2.1) using the fact that the initial forms of the generators of a minimal reduction of $I$ (which is a parameter ideal in $A$ ) form a regular sequence in $\operatorname{gr}_{A}(I)$.

Proposition (2.5). Let $(A, \mathfrak{m})$ be a Cohen-Macaulay ring of dimension $d$. Let $I$ be a strongly Cohen-Macaulay ideal in $A$. Assume $\mu\left(I_{\mathfrak{p}}\right) \leq \mathrm{ht}(\mathfrak{p})$ for all prime ideals $\mathfrak{p} \supseteq I$. Then $a\left(\operatorname{gr}_{A}(I)\right)=-\mathrm{ht}(I)$.

Proof. Put $s=\mathrm{ht}(I)=\operatorname{grade}(I)$, and $\mu(I)=s+t$. Note that the homology $H_{i}(K$.$) of the Koszul complex K$. of $A$ with respect to some minimal system of generators of $I$ is zero for $i>t$. Moreover $\operatorname{gr}_{A}(I)$ is $\mathrm{CM}$ and the $\mathscr{M}$-complex is exact by $\S 1,(5)$ and (7), which implies in particular that $I$ is of linear type. The $\mathscr{M}$-complex gives a resolution of $\operatorname{gr}_{A}(I)$ :

$$
0 \rightarrow \mathscr{M}_{t} \stackrel{\varphi_{t}}{\rightarrow} \cdots \rightarrow \mathscr{M}_{1} \stackrel{\varphi_{1}}{\rightarrow} \mathscr{M}_{0} \rightarrow \mathrm{gr}_{A}(I) \rightarrow 0
$$

where $\mathscr{M}_{i}=H_{i}\left(K_{\cdot}\right) \otimes A\left[X_{1}, \ldots, X_{s+t}\right], \operatorname{dim}\left(\mathscr{M}_{i}\right)=d+t$ and $\mathscr{M}_{i}$ is $\mathrm{CM}$ over $A\left[X_{1}, \ldots, X_{s+t}\right]$. The idea is now to compute $a:=a\left(\operatorname{gr}_{A}(I)\right)$ via the $a$-invariants $a\left(\mathscr{M}_{i}\right)$ : Applying Lemma $(2.1)$ exactly $(s+t)$ times we see that

$$
a\left(\mathscr{M}_{i}\right)=a\left(H_{i}(K \cdot)\right)-\mu(I)=-\mu(I),
$$

since (1) is considered as a sequence of $A\left[X_{1}, \ldots, X_{s+t}\right]$-modules. Note that by construction the morphism $\varphi_{i}$ are of degree 1 . Therefore we get the following exact sequences for the cokernels $\mathscr{D}_{i}$ of $\varphi_{i+1}$

$$
0 \rightarrow \mathscr{D}_{i+1}(-1) \rightarrow \mathscr{M}_{i} \rightarrow \mathscr{D}_{i} \rightarrow 0
$$

with morphisms of degree zero.

Since $G:=\operatorname{gr}_{A}(I)$ and $\mathscr{M}_{i}$ are CM, we get for the local cohomology with respect to the maximal homogeneous ideal $M$ of $A / I\left[X_{1}, \ldots, X_{s+t}\right]$

$$
H_{M}^{d}(G)_{j} \simeq H_{M}^{d+1}\left(\mathscr{D}_{1}\right)_{j-1} \simeq \cdots \simeq H_{M}^{d+t-1}\left(\mathscr{D}_{t-1}\right)_{j-t+1} .
$$

Moreover we have the exact sequence

$$
0 \rightarrow H_{M}^{d+t-1}\left(\mathscr{D}_{t-1}\right)_{j-t+1} \rightarrow H_{M}^{d+t}\left(\mathscr{D}_{t}\right)_{j-t} \rightarrow H_{M}^{d+t}\left(\mathscr{M}_{t-1}\right)_{j-t+1} .
$$

Note that

$$
H_{M}^{d+t}\left(\mathscr{D}_{t}\right)_{j-t} \simeq H_{M}^{d+t}\left(\mathscr{M}_{t}\right)_{j-t} .
$$

Case 1. $j>-h$, where $h=\mathrm{ht}(I)$, i.e. $j-t>-\mu(i)=:-n$. Therefore $H_{M}^{d+t}\left(\mathscr{M}_{t}\right)_{j-t}=0$, since $a\left(\mathscr{M}_{t}\right)=-n$. But by (3), (4) and (5) we know that

$$
H_{M}^{d}(G)_{j} \simeq H_{M}^{d+t-1}\left(\mathscr{D}_{t-1}\right)_{j-t+1} \subset H_{M}^{d+t}\left(\mathscr{M}_{t}\right)_{j-t}=0,
$$

hence $a(G) \leq-h$. 
Case 2. $j=-h$. Then (3) implies

$$
H_{M}^{d}(G)_{-h} \simeq H_{M}^{d+t-1}\left(\mathscr{D}_{t-1}\right)_{-h-t+1} \simeq H_{M}^{d+t}\left(\mathscr{M}_{t}\right)_{-n} \neq 0
$$

hence $a(G)=-\mathrm{ht}(I)$.

Remark. We can apply (2.5) in particular to an almost complete intersection ideal $I$, if $A / I$ is a Cohen-Macaulay ring (in this case $I$ is strongly CohenMacaulay). It is shown in [12] that the formula for the $a$-invariant in (2.5) holds for any (possibly non-Cohen-Macaulay) almost complete intersection ideal $I$.

Next we describe the relationship between the $a$-invariants $a\left(\operatorname{gr}_{A}(I)\right)$ and $a\left(\operatorname{gr}_{A}\left(I^{n}\right)\right)$. This result is a generalization of [9, Corollary 4] and it will be used in $\S 3$ for the characterization of the Gorenstein property of $R\left(I^{n}\right)$ and $\operatorname{gr}_{A}\left(I^{n}\right)$.

Proposition (2.6). Let $I$ be an ideal in a local ring $A$ and assume that $\operatorname{gr}_{A}(I)$ is Cohen-Macaulay. Then

$$
a\left(\operatorname{gr}_{A}\left(I^{n}\right)\right)=\left[a\left(\operatorname{gr}_{A}(I)\right) / n\right],
$$

where [ ] denotes the smallest integral part.

Proof. Put $d=\operatorname{dim}(A), a=a\left(\operatorname{gr}_{A}(I)\right), l=[a / n]$ and write $a=l n+r$ with $r \in\{0, \ldots, n-1\}$. For every $i \in\{1, \ldots, n\}$ there is an exact sequence of $R\left(I^{n}\right)$-modules:

$$
0 \rightarrow I^{n-i+1} \mathrm{gr}_{A}\left(I^{n}\right) \rightarrow I^{n-i} \mathrm{gr}_{A}\left(I^{n}\right) \rightarrow \operatorname{gr}_{A}(I)(n-i)^{(n)} \rightarrow 0
$$

Let $N$ be the maximal homogeneous ideal of $R\left(I^{n}\right)=R(I)^{(n)}$. Then (see [3, Proposition (47.5)]):

$$
H_{N}^{i}\left(\operatorname{gr}_{A}(I)(n-i)^{(n)}\right) \simeq H_{M}^{i}\left(\operatorname{gr}_{A}(I)(n-i)\right)^{(n)} .
$$

Hence for every $j \in Z$ and $i \in\{1, \ldots, n\}$ there is an exact sequence

$$
0 \rightarrow H_{N}^{d}\left(I^{n-i+1} \operatorname{gr}_{A}\left(I^{n}\right)\right)_{j} \rightarrow H_{N}^{d}\left(I^{n-i} \operatorname{gr}_{A}\left(I^{n}\right)\right)_{j} \rightarrow H_{M}^{d}\left(\operatorname{gr}_{A}(I)\right)_{n j+n-i} \rightarrow 0 .
$$

First consider these sequences for $j \geq l+1$. Since $n j+n-i \geq n(l+1)+$ $n-i>n l+r=a$, we have $H_{M}^{d}\left(\operatorname{gr}_{A}(I)\right)_{n j+n-i}=0$ for each $i \in\{1, \ldots, n\}$. Using the above sequences it follows inductively that $H_{N}^{d}\left(I^{n-i} \operatorname{gr}_{A}\left(I^{n}\right)\right)_{j}=0$ for $i=0, \ldots, n$. In particular $H_{N}^{d}\left(\operatorname{gr}_{A}\left(I^{n}\right)\right)_{j}=0$ for any $j \geq l+1$, i.e. $a\left(\operatorname{gr}_{A}\left(I^{n}\right)\right) \leq l$. To finish the proof, consider the cohomology sequences from above in degree $j=l$. Since $H_{M}^{d}\left(\operatorname{gr}_{A}(I)\right)_{n l+r} \neq 0$ we get $H_{N}^{d}\left(I^{r} \operatorname{gr}_{A}\left(I^{n}\right)\right)_{l} \neq$ 0 and then successively $H_{N}^{d}\left(I^{r-k} \operatorname{gr}_{A}\left(I^{n}\right)\right)_{l} \neq 0$ for $k=1, \ldots, r$. Hence $H_{N}^{d}\left(\operatorname{gr}_{A}\left(I^{n}\right)\right)_{l} \neq 0$. Q.E.D.

Corollary (2.7). Let $I$ be an ideal of height $\geq 1$ in a local ring $A$. If $\operatorname{gr}_{A}(I)$ is Cohen-Macaulay and $R\left(I^{n}\right)$ is Cohen-Macaulay for some $n \in N$, then $R(I)$ is Cohen-Macaulay.

Proof. First, note that

$$
\begin{aligned}
-1 & \geq a\left(\operatorname{gr}_{A}\left(I^{n}\right)\right) \quad \text { by }(2.2)(\mathrm{a}) \\
& =\left[a\left(\operatorname{gr}_{A}(I)\right) / n\right] \quad \text { by }(2.6) .
\end{aligned}
$$

Hence $a\left(\operatorname{gr}_{A}(I)\right)$ is negative. Finally, by $(2.2)(\mathrm{a}), R(I)$ is Cohen-Macaulay.

Remark. Note that in general the Cohen-Macaulayness of $R\left(I^{n}\right)$ does not imply this property for $R(I)$. 


\section{Powers of Strongly Cohen-Macaulay ideals}

We start with the following general observation.

Proposition (3.1). Let $I$ be an ideal of height $\geq 2$ in a Noetherian local ring $A$. Then at most one power of I has a Gorenstein Rees algebra.

Proof. Assume that $R\left(I^{s}\right)$ and $R\left(I^{t}\right)$ are Gorenstein. Since $R\left(I^{s t}\right)=R\left(I^{s}\right)^{(t)}=$ $R\left(I^{t}\right)^{(s)}$ and since $R\left(I^{s}\right)(-1)$ and $R\left(I^{t}\right)(-1)$ are canonical modules of $R\left(I^{s}\right)$ and $R\left(I^{t}\right)$ (see $(2.3)$ for the correct shifting degree -1 ) we know that both, $R\left(I^{s}\right)(-1)^{(t)}$ and $R\left(I^{t}\right)(-1)^{(s)}$ are canonical modules of $R\left(I^{s t}\right)$. Hence they must be isomorphic, and comparing their homogeneous parts of degree $j \geq 1$ we see that the ideals $I^{s(t j-1)}$ and $I^{t(s j-1)}$ are isomorphic as $A$-modules. By the following Lemma (3.2) we get $s=t$.

Lemma (3.2). Let $I$ be an ideal of height $\geq 2$ in a Noetherian ring $A$. If two powers $I^{s}$ and $I^{t}$ are isomorphic, then $s=t$.

Proof. We may assume that $A$ is a local ring with maximal ideal $\mathrm{m}$. The isomorphism $I^{s} \simeq I^{t}$ induces isomorphisms $I^{j s} / \mathrm{m} I^{j s} \simeq I^{j t} / \mathrm{m} I^{j t}$ for all numbers $j$. Now, there is a polynomial $P=\sum_{i=0}^{l-1} a_{i} X^{i} \in Q[X]$ of degree $l-1$ (where $l$ denotes the analytic spread of $I)$ such that $P(i)=\lambda\left(I^{i} / \mathrm{m} I^{i}\right)$ for $i \gg 0(\lambda \mathrm{de}-$ notes the length). From $P(s j)=P(t j)$ for $j \gg 0$ we get $a_{l-1} s^{l-1}=a_{l-1} t^{l-1}$. Since $l \geq \operatorname{ht}(I) \geq 2$, we get $s=t$.

For proving our main theorem, Theorem (3.5), we also need the following structure theorem for the canonical module of the Rees algebra. It comes from Corollary (2.5) in [7], if one takes the correct gradings in the proof given there.

Proposition (3.3) [7, Corollary (2.5)]. Let I be a proper ideal in a local ring $A$ of dimension $d$. Assume that $A$ is Gorenstein and $R(I)$ is Cohen-Macaulay. Then $\operatorname{gr}_{A}(I)$ is Gorenstein iff $(1, t)^{-a-2}(-1)$ is a canonical module of $R(I)$, where $a:=a\left(\operatorname{gr}_{A}(I)\right)$ and $(1, t)^{m}$ denotes the $R(I)$-submodule of the polynomial ring $A[t]$ which is generated by $1, t, \ldots, t^{m}$ in case $m \geq 0$ or $(1, t)^{-1}=I R(I)$ in case $m=-1$.

Next we need the following lemma:

Lemma (3.4). Assume that $A$ is Gorenstein and $R(I)$ is Cohen-Macaulay. If $(1, t)^{m}(-1)$ is a canonical module of $R(I)$ for some integer $m \geq-1$, then $R\left(I^{m+1}\right)$ is Gorenstein.

Proof. Note that for $m=-1$ there is nothing to prove. Hence we may assume $m \geq 0$. Then, denoting $(1, t)^{m}(-1)$ by $K$, we get

$$
K_{j}=(1, t)^{m}(-1)_{j}= \begin{cases}0 & \text { if } j \leq 0 \\ A & \text { if } 1 \leq j \leq m+1 \\ I^{j-(m+1)} & \text { if } j \geq m+2\end{cases}
$$

Now recall that the Veronesean $K^{(m+1)}$ is a canonical module of the Veronesean $R(I)^{(m+1)}=R\left(I^{m+1}\right)$. We get

$$
\left(K^{(m+1)}\right)_{j}=K_{j(m+1)}= \begin{cases}0 & \text { if } j \leq 0 \\ A & \text { if } j=1, \\ I^{j(m+1)-(m+1)} & \text { if } j \geq 2 .\end{cases}
$$


Hence $R\left(I^{m+1}\right)(-1)=K^{(m+1)}$, which proves the claim.

Here is the main theorem:

Theorem (3.5). Let $I$ be an ideal of height $\geq 2$ in the local ring A. Assume that $\operatorname{gr}_{A}(I)$ is Gorenstein. Then the following hold for $n \in N$ :

(a) $R\left(I^{n}\right)$ is Gorenstein iff $R(I)$ is Cohen-Macaulay and $n=-a\left(\operatorname{gr}_{A}(I)\right)-1$.

(b) If $R(I)$ is Cohen-Macaulay, then $\operatorname{gr}_{A}\left(I^{n}\right)$ is Gorenstein iff $-a\left(\operatorname{gr}_{A}(I)\right) \equiv 1$ $\bmod (n)$.

Proof. (a) Put $a=a\left(\operatorname{gr}_{A}(I)\right)$. Since $\operatorname{gr}_{A}(I)$ is Gorenstein and $R(I)$ is CohenMacaulay by $(2.7)$, we know by $(3.3)$ that $K:=(1, t)^{-a-2}(-1)$ is a canonical module of $R(I)$. It follows that $R\left(I^{-a-1}\right)$ is Gorenstein, by (3.4). This is by (3.1) the only power of $I$ which has a Gorenstein Rees algebra.

(b) Put $b=a\left(\operatorname{gr}_{A}\left(I^{n}\right)\right)$. We can assume that $n \geq 2$. Note that $R\left(I^{n}\right)$ and $\operatorname{gr}_{A}\left(I^{n}\right)$ are Cohen-Macaulay rings and that $K^{(n)}$ is a canonical module of $R(I)^{(n)} \simeq R\left(I^{n}\right)$. Hence by (3.3) $\mathrm{gr}_{A}\left(I^{n}\right)$ is Gorenstein if and only if the $R\left(I^{n}\right)$-module $L:=(1, t)^{-b-2}(-1)$ is isomorphic to $K^{(n)}$. Therefore, to finish the proof, we have to show that this statement holds iff $-a \equiv 1 \bmod (n)$. First we note that $b=[a / n]$ by (2.6), i.e. $a=b n+r$ with $r \in\{0, \ldots, n-1\}$. Hence we prove the following claim.

Claim. $L \simeq K^{(n)}$ iff $r=n-1$.

Assume that $r=n-1$. Then we get for each $j \in Z$ :

$$
L_{j}=(1, t)^{-b-2}(-1)_{j}= \begin{cases}0 & \text { if } j \leq 0 \\ A & \text { if } 1 \leq j \leq-b-1, \\ I^{n(j-1+b-2)} & \text { if } j \geq-b\end{cases}
$$

and

$$
\begin{aligned}
\left(K^{(n)}\right)_{j} & =\left((1, t)^{-a-2}(-1)^{(n)}\right)_{j}=\left((1, t)^{-b n-n-1}\right)_{j n-1}, \\
& = \begin{cases}0 & \text { if } j n-1<0, \\
A & \text { if } 0 \leq j n-1 \leq-b n-n-1, \\
I^{(j n-1)+(b n+n+1)} & \text { if } j n-1 \geq-b n-n,\end{cases} \\
& = \begin{cases}0 & \text { if } j \leq 0, \\
A & \text { if } 1 \leq j \leq-b-1, \\
I^{n(j+b+1)} & \text { if } j \geq-b-1+1 / n, \text { i.e., if } j \geq-b .\end{cases}
\end{aligned}
$$

Hence $L \simeq K^{(n)}$. For the converse assume that $L \simeq K^{(n)}$; in particular $L_{-b} \simeq$ $K_{-b n}=K_{r-a}$, i.e. $I^{n} \simeq I^{r+1}$. Since $\mathrm{ht}(I) \geq 2$ it follows $n=r+1$ by (3.2). This proves the claim and statement (b) of the theorem.

As immediate consequences of (3.5) we get the following propositions.

Proposition (3.6). Let $A$ be a Gorenstein local ring and I a strongly CohenMacaulay ideal satisfying $\mu\left(I_{\mathfrak{p}}\right) \leq \mathrm{ht}(\mathfrak{p})$ for all prime ideals $\mathfrak{p} \supset I$. Assume that $h:=\operatorname{ht}(I) \geq 2$. Then:

(a) $R\left(I^{n}\right)$ is Gorenstein iff $n=h-1$,

(b) $\operatorname{gr}_{A}\left(I^{n}\right)$ is Gorenstein iff $h \equiv 1 \bmod (n)$.

Proof. Use (3.5) together with (2.5) and $\S 1(7)$. 
Proposition (3.7). Let $(A, \mathfrak{m})$ be a d-dimensional Gorenstein local ring with reduction exponent $\delta(\mathfrak{m}) \leq 2$ and $d \geq 3$. Then the following hold for $n \in N$ :

(a) $\operatorname{gr}_{A}\left(\mathfrak{m}^{n}\right)$ is Gorenstein iff $d-\delta(\mathfrak{m}) \equiv 1 \bmod (n)$.

(b) $R\left(\mathfrak{m}^{n}\right)$ is Gorenstein iff $n=d-\delta(\mathfrak{m})-1$.

In particular: If $A$ is regular, then $R\left(\mathfrak{m}^{d-1}\right)$ is Gorenstein, and if $A$ is a quadratic hypersurface, then $R\left(\mathrm{~m}^{d-2}\right)$ is Gorenstein.

Proof. Use (3.5) together with (2.4)(a) and (b).

Remark (3.8). (a) We stated Propositions (3.6) and (3.7) as a direct consequence of Theorem (3.5). This theorem was shown by using the structure theorem (3.3). In the following we indicate another method for proving the "only if" parts in (3.6) and (3.7), which does not depend on (3.3).

Proof idea. Using the sequences in the proof of (2.6)

$$
0 \rightarrow I^{n-i+1} \mathrm{gr}_{A}\left(I^{n}\right) \rightarrow I^{n-i} \operatorname{gr}_{A}\left(I^{n}\right) \rightarrow \mathrm{gr}_{A}(I)(n-i)^{(n)} \rightarrow 0
$$

one can show the following: If $I$ is an ideal primary to the maximal ideal $\mathfrak{m}$ of a local ring $(A, \mathfrak{m})$, such that $\operatorname{gr}_{A}(I)$ and $\operatorname{gr}_{A}\left(I^{n}\right)$ are Gorenstein rings for some $n$, then $a\left(\mathrm{gr}_{A}(I)\right) \equiv-1 \bmod (n)$ (see [12]; the primary property of $I$ is used in order to know that the homogeneous parts of the graded local cohomology modules of the modules in the above sequences are of finite length). Hence, if $I$ is an ideal satisfying the conditions in (3.6), then by localizing at a minimal prime of $I$ we reach the described situation (i.e. the primary case). Since moreover $a\left(\operatorname{gr}_{A}(I)\right)=-\mathrm{ht}(I)=a\left(\operatorname{gr}_{A_{\mathfrak{p}}}\left(I_{\mathfrak{p}}\right)\right)$ for $\mathfrak{p} \in \operatorname{Assh}_{A}(A / I)$ (by (2.5)), the implication " $\mathrm{gr}_{A}\left(I^{n}\right)$ Gorenstein $\Rightarrow h \equiv 1 \bmod (n)$ " follows. Finally (under the assumptions of (3.6)) the Gorenstein property of $R\left(I^{n}\right)$ forces $\operatorname{gr}_{A}\left(I^{n}\right)$ to be Gorenstein too, by (2.2); hence $h \equiv 1 \bmod (n)$, as we have just pointed out. Furthermore $-2=a\left(\operatorname{gr}_{A}\left(I^{n}\right)\right)=[-h / n]$ by $(2.2),(2.6)$ and (2.5). Now it is easy to see that the two relations $h \equiv 1 \bmod (n)$ and $[-h / n]=-2$ have the unique common solution $n=h-1$.

Similar arguments can be used to prove the "only if" parts in (3.7).

(b) In (3.7), also the "if" parts can be shown without using (3.3). If $A$ is regular or a quadratic hypersurface, it comes out by elementary computations that for the special numbers $n$ mentioned in (3.7) the form rings $\mathrm{gr}_{A}\left(\mathfrak{m}^{n}\right)$ are Gorenstein rings, see [12].

Remark (3.9). The assumption $d \geq 3$ in Proposition (3.7) was only used to get that $R(I)$ is Cohen-Macaulay. Then one could apply Theorem (3.5)(b). From the arguments given in (3.8)(a) it is easy to see that Proposition (3.7) is also true for $d=2$.

\section{Powers of EQUimultiple IDEALS}

Part I: m-primary ideals. Next we show to which extent the Gorensteinness of $R\left(I^{n}\right)$, where $I$ is m-primary, determines the structure of the ideal $I$ via the reduction exponent $\delta(I)$. As a corollary we obtain a characterization of regular $d$-dimensional local rings $(A, \mathfrak{m})$ by the Gorensteinness of $R\left(\mathfrak{m}^{d-1}\right)$, which is a generalization of a result of Goto-Shimoda [1, Proposition 4.8]. First we prove the Key-lemma:

Key-lemma (4.1). Let $I$ be an m-primary ideal in the d-dimensional local ring $(A, \mathfrak{m})$, and $q=\left(x_{1}, \ldots, x_{l}\right)$ a minimal reduction of $I$. Assume that $\operatorname{gr}_{A}\left(I^{n}\right)$ 
is Cohen-Macaulay for some natural number $n$. Put $a:=a\left(\operatorname{gr}_{A}\left(I^{n}\right)\right)$. Then $I^{n a+n+d} \subseteq q$. If moreover $R\left(I^{n}\right)$ is Gorenstein and $d \geq 2$, then $I^{d-n} \subseteq q$, hence $n<d$.

Corollary (4.2). Let I be an $\mathfrak{m}$-primary ideal in a Cohen-Macaulay ring $A$ with dimension $d \geq 2$. If $R\left(I^{d-1}\right)$ is Gorenstein, then $I$ is a parameter ideal.

Proof of Lemma (4.1). Clearly, $J:=\left(x_{1}^{n}, \ldots, x_{d}^{n}\right)$ is a minimal reduction of $I^{n}$, and the ideal $J^{*}$ generated by the initial forms $\left(x_{i}^{n}\right)^{*}$ in $\operatorname{gr}_{A}\left(I^{n}\right)$ is a complete intersection in the Cohen-Macaulay ring $\operatorname{gr}_{A}\left(I^{n}\right)$. Since $G:=\operatorname{gr}_{A}\left(I^{n}\right) / J^{*}$ is artinian and $a(G)=a+d$, we get $G_{a+d+1}=0$. That means

$$
I^{n(a+d+1)}=I^{n(a+d)} J \subseteq J .
$$

Since $x_{1}, \ldots, x_{d}$ form a regular sequence we get

$$
I^{n a+n+d}=I^{n(a+d+1)-d(n-1)} \subseteq q .
$$

If moreover $R\left(I^{n}\right)$ is Gorenstein, then $a=-2$ by (2.2)(b), hence $I^{d-n} \subseteq q$. As a consequence we obtain

Proposition (4.3). An equimultiple ideal I of height $h \geq 2$ in a Gorenstein local ring $A$ is a complete intersection if and only if $R\left(I^{h-1}\right)$ is Gorenstein.

Proof. By Proposition (3.6) the "only if" part is already clear. For the converse let $q$ be a minimal reduction of $I$. Since $I$ is equimultiple $q$ is generated by a regular sequence. On the other hand, by Corollary (4.2) $I A_{\mathfrak{p}}=q A_{\mathfrak{p}}$ for all $\mathfrak{p} \in \operatorname{Assh}(A / I)$, thus we have $I=q$ since $\operatorname{Ass}(I / q) \subseteq \operatorname{Ass}(A / q)=\operatorname{Assh}(A / q)=$ $\operatorname{Assh}(A / I)$ and hence $\operatorname{Ass}(I / q)$ is empty.

Theorem (4.4). Given a d-dimensional local Gorenstein ring $(A, \mathfrak{m})$ and an integer $i \in\{1,2,3\}$. Then for $d-i \geq 1$ we have

$$
R\left(\mathfrak{m}^{d-i}\right) \text { is Gorenstein iff } \delta(\mathfrak{m})=i-1 .
$$

Proof. If $\delta(\mathfrak{m})=i-1$ then $R\left(\mathfrak{m}^{n}\right)$ is Gorenstein for $n=d-\delta(\mathfrak{m})-1=d-i$ by (3.7). Conversely, assume that $R\left(\mathrm{~m}^{d-i}\right)$ is Gorenstein and let $q$ be a minimal reduction of $\mathfrak{m}$. By (4.1), we know that $\mathfrak{m}^{i} \subseteq q$. Hence, for $i \in\{1,2\}$ we get $\mathfrak{m}^{i}=q \mathfrak{m}^{i-1}$ and $\mathfrak{m}^{i-1} \neq q \mathfrak{m}^{i-2}$ by (3.1) and (3.7), i.e. $\delta(\mathfrak{m})=i-1$ for these two cases. For $i=3$ assume that $R\left(\mathrm{~m}^{d-3}\right)$ is Gorenstein. Then $m^{3} \subseteq q$, where $q$ is a minimal reduction of $\mathfrak{m}$. In particular $\mathfrak{m}^{3} \subseteq q \mathfrak{m}$, and since $A$ is Gorenstein $\mathfrak{m}^{3}=q \mathfrak{m}^{2}$ by [13, Proposition (3.3) and Theorem (3.4)]. From this we get $\delta(\mathfrak{m})=2$ since $\delta(\mathfrak{m}) \notin\{0,1\}$ again by (3.1) and (3.7).

Corollary (4.5). Let $(A, \mathfrak{m})$ be a d-dimensional Cohen-Macaulay local ring. Then:

(a) $R\left(\mathfrak{m}^{d-1}\right)$ is Gorenstein iff $A$ is regular.

(b) $R\left(\mathfrak{m}^{d-2}\right)$ is Gorenstein iff $A$ is a hypersurface with $e(A)=2$.

(c) $R\left(\mathfrak{m}^{d-3}\right)$ is Gorenstein iff $A$ is Gorenstein and $\operatorname{emb}(A)=e(A)+d-2$, where $d \geq 2$ for (a), $d \geq 3$ for (b), and $d \geq 4$ for (c).

Remark. Corollary (4.5) was also proved by A. Ooishi [11, Proposition (4.6)]. Moreover he could show that if $e=e(A)<d, R\left(\mathfrak{m}^{d-e}\right)$ is Gorenstein if and only if $A$ is a hypersurface [11, Proposition (4.7)]. In the following we prove a somewhat similar result, which contains the "if part" of Ooishi's result. 
Proposition (4.6). Let $(A, \mathfrak{m})$ be a hypersurface with $d=\operatorname{dim}(A) \geq 2$. Then $R\left(\mathfrak{m}^{n}\right)$ is Gorenstein iff $d>e$ and $n=d-e$.

Proof. Since $A$ is a hypersurface, $\operatorname{gr}_{A}(\mathfrak{m})$ is Gorenstein. We put $a=a\left(\operatorname{gr}_{A}(\mathfrak{m})\right)$. Then $R\left(\mathfrak{m}^{n}\right)$ is Gorenstein $\Leftrightarrow R(\mathfrak{m})$ is Cohen-Macaulay and

$$
\begin{aligned}
& n=-a-1 \quad(\text { by Theorem }(3.5)) \\
\Leftrightarrow & a<0 \text { and } n=-a-1 \\
\Leftrightarrow & e \leq d \text { and } n=d-e \quad(\text { since } a=\delta(\mathfrak{m})-d=e-1-d) \\
\Leftrightarrow & n=d-e(\geq 1) .
\end{aligned}
$$

If $\operatorname{gr}_{A}(\mathfrak{m})$ is Cohen-Macaulay, but $A$ is not a hypersurface, then $\delta(\mathfrak{m}) \leq$ $e-2$. The next statement describes the case $\delta(\mathfrak{m})=e-2$.

Proposition (4.7). Let $(A, \mathfrak{m})$ be a d-dimensional local Cohen-Macaulay ring with $d \geq 2$. Assume that

(i) $R(\mathfrak{m})$ is Gorenstein,

(ii) $\delta(\mathfrak{m})=e-2$.

Then $e=d=4$.

Proof. From assumption (i) we get by [8, Corollary (3.6)(2)] that $\delta(\mathfrak{m})=d-2$, hence $d=e$ by assumption (ii). Then [2, Theorem (4.1)(iii)] implies that $d=4$.

Part II: Equimultiple prime ideals. First we mention two known results about the Gorenstein property of the Rees ring of equimultiple ideals of height 2.

Proposition (4.8) [2, Theorem 2.6]. Let $A$ be generalized Cohen-Macaulay ring of dimension $\geq 4$ and $\mathfrak{p}$ an equimultiple prime ideal of height 2 . If $R(\mathfrak{p})$ is Gorenstein, then $A$ is a Gorenstein-domain and $\mathfrak{p}$ is a complete intersection.

Proposition (4.9) [2, Proposition 4.10]. Let $A$ be a local ring of dimension $\geq 3$ and $\mathfrak{p}$ a prime ideal of height 2 such that

(i) $R(\mathfrak{p})$ is Gorenstein,

(ii) $\mathfrak{p} / \mathfrak{p}^{2}$ is a free $A / \mathfrak{p}$-module.

Then $A$ is a Gorenstein-domain and $\mathfrak{p}$ is a complete intersection.

Remark. (a) Note that $\mathfrak{p}$ in (4.9) is equimultiple. (b) The crucial step in the proofs of (4.8) and (4.9) was to show that $A$ is Cohen-Macaulay. Then $A$ was even Gorenstein by $(2.2)(\mathrm{b})$ and $\mathfrak{p}$ is a complete intersection by [8, (3.6)]. Hence $R(\mathfrak{p})$ was Cohen-Macaulay. Finally the domain property of $A$ followed from the fact that $A_{\mathfrak{p}}$ was regular; see [2, (2.1)].

In the following we give generalizations of (4.8) and (4.9).

Proposition (4.10). Let $A$ be a d-dimensional generalized Cohen-Macaulay ring and $\mathfrak{p}$ an equimultiple prime ideal of height $h \geq 2$. If $R\left(\mathfrak{p}^{h-1}\right)$ is Gorenstein and $d \geq 2 h$, then $A$ is a Gorenstein-domain and $\mathfrak{p}$ is a complete intersection.

Proposition (4.11). Let $(A, \mathfrak{m})$ be $a d$-dimensional local ring and $\mathfrak{p} \neq \mathfrak{m} a$ prime ideal of height $h \geq 2$ such that $A / \mathfrak{p}$ and $A_{\mathfrak{p}}$ are Cohen-Macaulay.

Assume that

(i) $R\left(\mathfrak{p}^{h-1}\right)$ is Gorenstein,

(ii) $\mathfrak{p} / \mathfrak{p}^{2}$ is a free $A / \mathfrak{p}$-module. 
Then $A$ is a Gorenstein-domain and $\mathfrak{p}$ is a complete intersection.

Proof of (4.10) and (4.11). For both propositions it is enough to show that $A$ is Cohen-Macaulay. If this is known, $A$ must be Gorenstein by $(2.2)(b)$ and $\mathfrak{p}$ is a complete intersection by (4.3). Since $A_{\mathfrak{p}}$ is regular by (4.5) and $R(\mathfrak{p})$ is Cohen-Macaulay, $A$ is again a domain. For the Cohen-Macaulayness of $A$ in (4.11) we refer to the proof of (2.6) in [2].

The Cohen-Macaulay property of $A$ in (4.10) we get as follows: From the assumptions we conclude by [3, Proposition (4.5.4)(ii)] that $\operatorname{depth}(A) \geq$ $\operatorname{dim}(A / \mathfrak{p})+1$, i.e. $\operatorname{depth}(A) \geq d-h+1 \geq h+1$ because $d \geq 2 h$ by assumption. Hence $A \simeq K_{A}$ satisfies Serre's condition $S_{h+1}$, where $K_{A}$ is the canonical module of $A$. Then by [14, Satz 3.2.3] we know that $H_{\mathfrak{m}}^{i}(A)=0$ for $d-(h+1)+2 \leq i<d$, therefore $A$ is Cohen-Macaulay.

Example [8, Example (2)]. Let $A=K\left[\left[X_{1}, X_{2}, X_{3}, Y_{1}, Y_{2}, Y_{3}, Y_{4}\right]\right] / J$, where $K$ is a field of characteristic 2 and

$$
\begin{aligned}
J=\left(X_{1} Y_{1}+X_{2} Y_{2}+X_{3} Y_{3}, Y_{1}^{2},\right. & Y_{2}^{2}, Y_{3}^{2}, Y_{4}^{2}, Y_{1} Y_{4}, Y_{2} Y_{4}, Y_{3} Y_{4}, \\
& \left.Y_{1} Y_{2}-X_{3} Y_{4}, Y_{2} Y_{3}-X_{1} Y_{4}, Y_{1} Y_{3}-X_{2} Y_{4}\right) .
\end{aligned}
$$

$A$ is generalized Cohen-Macaulay. Let $\mathfrak{p}$ be the maximal ideal of $A$. Then

$$
h=: \operatorname{ht}(\mathfrak{p})=l(\mathfrak{p})=3 \text { and } d=3 \ngtr 2 h,
$$

$R(\mathfrak{p})$ is Gorenstein, i.e. $R\left(\mathfrak{p}^{2}\right)$ cannot be Gorenstein. Hence, two assumptions of (4.10) are not fulfilled, and we see that $A$ is not Cohen-Macaulay (otherwise, $A$ would be a hypersurface by (4.5), which is obviously not the case).

\section{ACKNOWLEDGMENTS}

The last author was supported by the DAAD (Germany) and DGICYT-grants BE 90-049 and PB 88-0224 (Spain). He received stimulating hospitality by the Mathematical Institute of the University of Cologne (Germany).

\section{REFERENCES}

1. S. Goto and Y. Shimoda, On the Rees algebras of Cohen-Macaulay rings, Commutative Algebra: Analytical Methods (R. N. Draper, ed.), Lecture Notes in Pure and Appl. Math., vol. 68, Dekker, New York and Basel, 1982, pp. 201-231.

2. M. Herrmann and S. Ikeda, On the Gorenstein property of Rees algebras, Manuscripta Math. 59 (1987), 471-490.

3. M. Herrmann, S. Ikeda, and U. Orbanz, Equimultiplicity and blowing up, Springer-Verlag, Berlin and Heidelberg, 1988.

4. J. Herzog, A. Simis, and W. V. Vasconcelos, Koszul homology and blowing up rings, Commutative Algebra, Proc. Trento Conf. (S. Greco and G. Valla, eds.), Lecture Notes in Pure and Appl. Math., vol 84, Dekker, New York and Basel 1983, pp. 79-169.

5. __ Approximation complexes of blowing up rings, J. Algebra 74 (1982), 466-493.

6. __ Approximation complexes of blowing up rings. II, J. Algebra 82 (1983), 53-83.

7. _ On the canonical module of the Rees algebra and the associated graded ring of an ideal, J. Algebra 105 (1987), 285-302.

8. S. Ikeda, On the Gorensteinness of Rees algebras over local rings, Nagoya Math. J. 102 (1986), 135-154.

9. T. Marley, The coefficients of the Hilbert polynomial and the reduction number of an ideal, J. London Math. Soc. (2) 40 (1989), 1-8. 
10. A. Ooishi, Stable ideals in Gorenstein local rings, J. Pure Appl. Algebra 69 (1990), 185-191.

11. $\ldots$ On the Gorenstein property of the associated graded ring and the Rees algebra of an ideal, preprint, 1990.

12. J. Ribbe, Thesis, Universität zu Köln, 1991.

13. J. Sally, Tangent cones at Gorenstein singularities, Compositio Math. (2) 40 (1980), 167175.

14. P. Schenzel, Dualisierende Komplexe in der lokalen Algebra und Buchsbaum-Ringe, Lecture Notes in Math., vol. 907, Springer-Verlag, 1982.

15. N. V. Trung and S. Ikeda, When is the Rees algebra Cohen-Macaulay?, Comm. Algebra 17 (1989), 2893-2922.

16. A. Lascu and M. Fiorentini, Linkage among subcanonical and quasicomplete intersection projective schemes, preprint, 1991.

(M. Herrmann and J. Ribbe) Mathematisches Institut der Universität Zu Köln, WeyerTAL 86-90, D-50931 KölN, GermaNY

(S. Zarzuela) Departament d’Àlgebra i Geometria, Universitat de Barcelona, Gran Via 585, E-08007 Barcelona, Spain

E-mail address: ribbe@mi.uni-koeln.de 